\title{
Variant classification changes over time in BRCA1 and BRCA2
}

\author{
Chloe Mighton, BSc ${ }^{1,2}$, George S. Charames, PhD FACMG ${ }^{3,4,5}$, Marina Wang, $\mathrm{MD}^{4}$, \\ Kathleen-Rose Zakoor, MBinf ${ }^{4,5}$, Andrew Wong, MSC ${ }^{4}$, Salma Shickh, MS CGC ${ }^{1,2}$, \\ Nicholas Watkins, MSc CGC/CCGC ${ }^{4}$, Matthew S. Lebo, PhD FACMG 6,7 , Yvonne Bombard, PhD ${ }^{1,2}$ and \\ Jordan Lerner-Ellis, PhD FACMG ${ }^{3,4,5}$
}

\begin{abstract}
Purpose: To report BRCA1 and BRCA2 (BRCA1/2) variant reassessments and reclassifications between 2012 and 2017 at the Advanced Molecular Diagnostics Laboratory (AMDL) in Toronto, Canada, which provides BRCA1/2 testing for patients in Ontario, and to compare AMDL variant classifications with submissions in ClinVar.
\end{abstract}

Methods: Variants were assessed using a standardized variant assessment tool based on the American College of Medical Genetics and Genomics/Association for Molecular Pathology's guidelines and tracked in an in-house database. Variants were shared through the Canadian Open Genetics Repository and submitted to ClinVar for comparison against other laboratories.

Results: AMDL identified 1209 BRCA1/2 variants between 2012 and 2017. During this period, $32.9 \%$ (398/1209) of variants were reassessed and $12.4 \%(150 / 1209)$ were reclassified. The majority of reclassified variants were downgraded (112/150, 74.7\%). Of the reclassified variants, $63.3 \%(95 / 150)$ were reclassified to benign, $20.7 \%(31 / 150)$ to likely benign, $10.0 \%(15 / 150)$ to variant of uncertain significance, $2.0 \%(3 / 150)$ to likely pathogenic, and $4.0 \%$ $(6 / 150)$ to pathogenic. Discordant ClinVar submissions were found for $40.4 \%(488 / 1209)$ of variants.

Conclusion: BRCA1/2 variants may be reclassified over time. Reclassification presents ethical and practical challenges related to recontacting patients. Data sharing is essential to improve variant interpretation, to help patients receive appropriate care based on their genetic results.

Genetics in Medicine (2019) 21:2248-2254; https://doi.org/10.1038/s41436019-0493-2

Keywords: genetic testing; variant reclassification; $B R C A 1$; BRCA2

\section{INTRODUCTION}

Accurate variant interpretation is essential for appropriate disease diagnosis and management through genetic testing. Variant interpretation involves the synthesis of multiple lines of evidence to determine a variant's pathogenicity. ${ }^{1}$ Determining whether a variant is clinically meaningful can be difficult ${ }^{1}$ as many rare and novel variants may not contribute to disease or may not be as rare as once thought. ${ }^{2}$ For many variants, there may be limited data or relevant evidence that is siloed within individual laboratories. ${ }^{3}$ Reassessment may result in changes in variant classification as new evidence becomes available, which can cause discrepancies between laboratories' interpretations if reassessment occurs at different intervals. ${ }^{4}$

Until recently, laboratories relied on site-specific methods to assess variant pathogenicity. ${ }^{1}$ Discrepancies existed in how laboratories weighted and assessed evidence, ${ }^{5}$ leading to discordant accounts of pathogenicity within and across databases that curate periodically or at a single time point. ${ }^{3}$ The American College of Medical Genetics and Genomics (ACMG) and the Association for Molecular Pathology (AMP) established guidelines for variant interpretation, ${ }^{6}$ but differences in interpretation continue to be a major challenge. ${ }^{7,8}$ Even variants in well-characterized genes, such as BRCA1 and BRCA2 (BRCA1/2), may differ in classification between laboratories. One study found discordance among BRCA1/2 variant classifications across several public databases. ${ }^{9}$ Others, however, have found high rates of concordance among $B R C A 1 / 2$ entries in ClinVar, ${ }^{3,10}$ with older entries more prone to discordance. $^{3}$

Variants of uncertain significance are important targets for reassessment. A variant of uncertain significance (VUS) can introduce ambiguity into a patient's care as it results in an inconclusive report. VUSs also have either the least evidence or most conflicting evidence and are thus

\footnotetext{
${ }^{1}$ Institute of Health Policy, Management and Evaluation, University of Toronto, Toronto, ON, Canada; ${ }^{2}$ Li Ka Shing Knowledge Institute, St. Michael's Hospital, Toronto, ON, Canada; ${ }^{3}$ Department of Pathology and Laboratory Medicine, University of Toronto, Toronto, ON, Canada; ${ }^{4}$ Mount Sinai Hospital, Laboratory Medicine and Pathobiology, Sinai Health System, University of Toronto, Toronto, ON, Canada; ${ }^{5}$ Lunenfeld-Tanenbaum Research Institute, Sinai Health System, Toronto, ON, Canada; ${ }^{6}$ Laboratory for Molecular Medicine Cambridge, Partners Healthcare for Personalized Medicine, Cambridge, MA, USA; ${ }^{7}$ Brigham and Women's Hospital and Harvard Medical School, Boston, MA, USA. Correspondence: Jordan Lerner-Ellis (Jordan.Lerner-Ellis@sinaihealthsystem.ca)
} 
likely to be reclassified as information changes. Clinical management recommendations differ for patients who harbor pathogenic variants and those with VUSs. A pathogenic mutation in BRCA1/2 confers a $45-65 \%$ risk for breast cancer by age $70,{ }^{11-13}$ and $10-39 \%$ risk for ovarian cancer by age $70 .{ }^{11}$ In Ontario, individuals with a pathogenic variant in BRCA1 or BRCA2 or who are at a greater than or equal to $25 \%$ risk of breast cancer assessed using IBIS or BOADICEA tools are eligible and recommended for screening with annual magnetic resonance image (MRI) and mammography through the Ontario Breast Screening Program. Alternatively, patients may elect to undergo mastectomy or salpingooophorectomy to reduce cancer occurrence or recurrence risk. These recommendations typically do not apply to patients with a VUS, who are managed based on personal and family history. ${ }^{14}$ However, some patients with VUSs have been found to pursue prophylactic surgeries. ${ }^{15}$ In two cases, it was found that the patient's VUS was reclassified to benign following the patients' prophylactic salpingo-oopherectomy, surgery that would not have been recommended for a patient with a benign variant. ${ }^{15}$

In Ontario, $B R C A 1 / 2$ testing is publicly funded by the provincial Ministry of Health and Long-term Care (MOHLTC) and performed at seven laboratories. ${ }^{16}$ The MOHLTC established 13 eligibility criteria, at least one of which must be met for an individual to be eligible for genetic testing for BRCA1/2. ${ }^{17}$ The Advanced Molecular Diagnostics Laboratory (AMDL) at Mount Sinai Hospital in Toronto has been providing publicly funded $B R C A 1 / 2$ testing for the province of Ontario since 2001. ${ }^{16}$ Among patients that met MOHLTC criteria and were tested at AMDL between 2007 and 2014, 16.2\% were found to have a pathogenic variant and $15.8 \%$ were found to have VUSs, likely pathogenic, or likely benign variants. ${ }^{16}$

Here, we report data on the number and type of variant reclassifications observed at AMDL between January 2012 and August 2017, and the average time between reclassification events. Additionally, we report a comparison of AMDL variant classifications to submissions from ClinVar.

\section{Patient population}

\section{MATERIALS AND METHODS}

Between 1 February 2001 and 18 August 2017, blood samples from patients at hereditary cancer clinics in Ontario were submitted to AMDL for BRCA1/2 testing. Samples were also received from Quebec between 2012 and 2014. All patients who received testing met at least 1 of the 13 eligibility criteria for BRCA1/2 testing established by the Ontario MOHLTC. ${ }^{17}$ This study was approved by the Research Ethics Board of Mount Sinai Hospital.

\section{Genetic testing}

Patients received one of three types of genetic testing: (1) full sequence analysis for BRCA1 [NM_007294.3] and BRCA2 [NM_000059.3] with deletion and duplication analysis, (2) analysis of three common Ashkenazi Jewish variants, or (3) analysis of a familial or known variant. The MOHLTC criteria establish patients' eligibility for each of these three options. If option 2 or 3 yielded a negative result, the referring clinician could request that their patient receive option 1 if they met criteria. The coding region and splice sites, $+/-15$ bp from the exon junction, were analyzed for variants by Sanger sequencing on an ABI 3730 DNA analyzer (ThermoFisher Scientific, https://www.thermofisher. com/order/catalog/product/3730S) and/or deletion, duplication analysis by multiplex ligation-dependent probe amplification (MLPA; MRC-Holland, www.mlpa.com). As of 2014-2017 DNA sequencing was performed by nextgeneration sequencing (NGS) on an Illumina MiSeq or NextSeq instrument following enrichment using the TruSight Rapid Capture Kit (Illumina) for the full coding regions and splice sites ( + or -15 base pairs from the exon boundaries) with a minimum coverage equal to or greater than $20 x$.

\section{Variant analysis and reporting}

Prior to 2011, variants identified at AMDL were classified based primarily on the expertise of the laboratory director signing out each case. Standardized, written protocols had not yet been established to guide classification, although the Breast Information Core (BIC) resource (https://research. nhgri.nih.gov/bic/) was used and other existing criteria may have been applied.

In 2011, a formal variant assessment tool (VAT) was introduced. The VAT was modified from a protocol codeveloped at Partners Healthcare in Boston, ${ }^{18,19}$ and aligned with the 2007 ACMG guidelines. ${ }^{20}$ The VAT was updated in October 2014 to include the Exome Aggregation Consortium (ExAC, exac.broadinstitute.org) database following its release. In May 2015, the VAT was revised based on the 2015 ACMG/AMP guidelines ${ }^{6}$ for variant assessment. Based on these criteria, variants are assigned one of five classifications: benign, likely benign, uncertain significance, likely pathogenic, or pathogenic. Variant classification using the VAT draws on evidence from multiple sources including primary literature, in silico and computational analyses, variant databases, publicly accessible population control databases, and the AMDL internal database.

\section{Variant reassessment}

Reassessment was conducted at the request of the ordering clinician, or if a variant was identified in a new proband and had not since been reclassified using the VAT. Typically, VUSs were reassessed when the variant was identified in a new family and more than a year had elapsed since the variant was last classified. Likely benign, benign, likely pathogenic, and pathogenic variants may also have been re-evaluated in this context based on clinical suspicion, but this occurred more rarely.

Variant reassessment involved the incorporation of new data, if available, and re-examination of the whole body of evidence regarding the variant. In July 2013, AMDL joined the Canadian Open Genetics Repository (COGR), a nationwide data sharing project, ${ }^{19}$ and variants were reassessed 
through a discrepancy-reporting and consensus-building process starting in May 2016. In some cases, reassessment resulted in a change to variant classification.

Changes to variant classification and interpretation evidence were tracked in an in-house database created in January 2012. When a variant was first identified, it was entered into the database with its initial classification, interpretation text, and linked to the patient report. The total number of reports and families with a particular variant was tracked and could be queried. Upon reassessment, the report sign-out date, date of reclassification, and updated classification were tracked in the database.

\section{Comparison to ClinVar submissions}

Variants were submitted to ClinVar. ${ }^{21}$ ClinVar generates a monthly conflict report, which can be accessed on the ClinVar FTP site (ftp://ftp.ncbi.nlm.nih.gov/pub/clinvar/). The report displays pairwise comparisons of discordant submissions. A conflict report (available on ClinVar FTP as summary_conflicting_interpretations.txt) was downloaded in July 2018 and used to quantify discordant submissions for the variants in the AMDL database. In addition, summary variant data (available on ClinVar FTP as variant_summary.txt) was downloaded from ClinVar FTP to obtain the overall ClinVar classification for each variant seen by AMDL.

\section{RESULTS}

Between January 2012 and August 2017, 6090 patients were seen at AMDL (Fig. 1). As of 1 January 2012, the AMDL database contained $586 B R C A 1 / 2$ variants. This grew by an average of 104 variants per year. By August 2017, there were 1209 unique variants in the AMDL database (Supplementary Appendix 1): 473 in BRCA1 and 736 in BRCA2.

\section{Variant reassessment and reclassification}

Between 2012 and August 2017, 32.9\% (398/1209) of the variants in the database were reassessed and $12.4 \%(150 / 1209)$ were reclassified. Reclassification occurred based on the availability of new evidence, such as population frequency from ExAC or the Genome Aggregation Database (gnomAD,

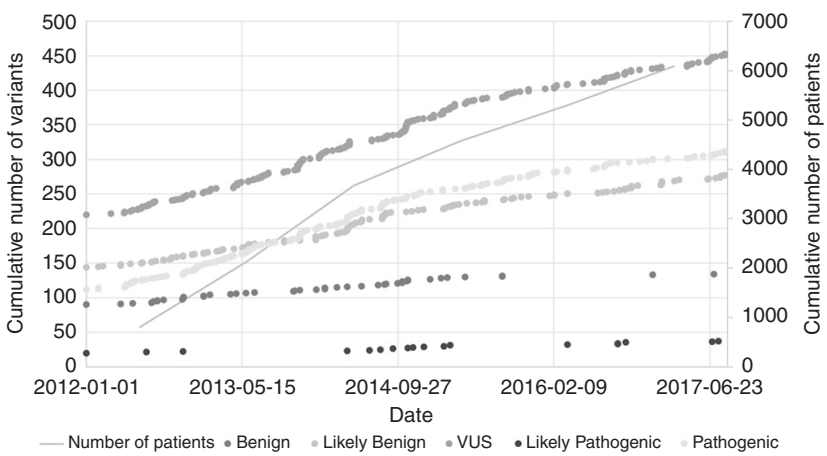

Fig. 1 Cumulative number of unique variants by initial classification, and number of patients tested by the Advanced Molecular Diagnostics Laboratory (AMDL) between 2012 and August 2017. VUS variant of uncertain significance. gnomad.broadinstitute.org); new publications with segregation or functional studies; or if the variant was identified as co-occurring with a pathogenic variant. Classifications, dates, and supporting evidence for reclassified variants are reported in Supplementary Appendix 1.

Of the 150 variants that were reclassified, $83.3 \%(125 / 150)$ were reclassified once, $16.0 \%(24 / 150)$ were reclassified twice, and $0.67 \%(1 / 150)$ reclassified three times. The majority of reclassified variants were downgraded (112/150, 74.7\%). Of the reclassified variants, $63.3 \%(95 / 150)$ were reclassified to a final classification of benign, $20.7 \%(31 / 150)$ to likely benign, $10.0 \%(15 / 150)$ to VUS, $2.0 \%(3 / 150)$ to likely pathogenic, and $4.0 \%(6 / 150)$ to pathogenic (Fig. 2). In $16.7 \%(25 / 150)$ of cases, the variant was reclassified more than once, and $8.0 \%$ $(12 / 150)$ of the reclassified variants were returned to their initial classification through two reclassification events (2 pathogenic, 1 likely benign, 9 benign). Figure 2 illustrates the final and initial classification categories for reclassified variants.

There were 453 variants initially classified as VUSs. Overall, $34.0 \%(154 / 453)$ of VUSs were reassessed and $14.8 \%(67 / 453)$ reclassified. Of the 67 VUSs that were reclassified, $61.2 \%$ (41/ $67)$ were reclassified to benign, $34.3 \%(23 / 67)$ to likely benign, $3.0 \%(2 / 67)$ to likely pathogenic, and $1.5 \%(1 / 67)$ to pathogenic. The remaining VUSs were not reassessed because they did not meet the laboratory's criteria for reassessment; i.e., they were not identified in another family more than one year after their initial identification, and no ordering clinicians requested reassessment. This reflects current practice in a publicly funded health care system laboratory without resources to routinely reassess all variants.

One variant of interest, $B R C A 2$ c.68-7T $>A$, was reclassified from pathogenic to benign over multiple reclassification events. This variant was first identified and classified as pathogenic in May 2010. The variant was shown to segregate with breast cancer in two other affected individuals in the family. Without contradictory data or structured standardized variant interpretation processes at that time, this evidence

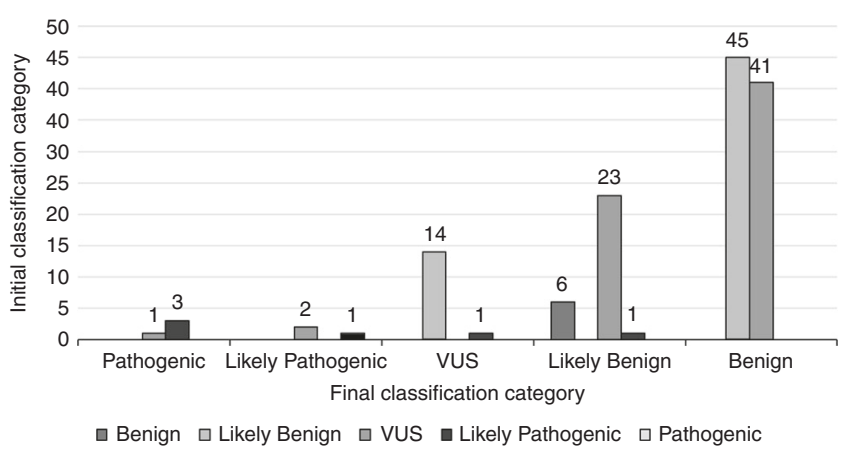

Fig. 2 Variant reclassifications. Number of variants that were reclassified between January 2012 and August 2017. The $x$-axis indicates the variant's final classification category, and the color of the bar indicates the variant's initial classification category. The 12 variants that were reclassified and returned to their initial classifications through multiple reclassification events are not reflected in this figure. VUS variant of uncertain significance. 
resulted in classification as pathogenic. In 2011, prior to the establishment of the variant tracking database, the variant was reclassified as a VUS (therefore, in our count of variant reclassifications between 2012 and 2017, this variant is counted as a change from VUS, not from pathogenic). The variant was reassessed in October 2014 following the release of the ExAC database, where it was observed with an overall frequency of 0.002 and reclassified to likely benign. ${ }^{22}$ The variant was also identified at a frequency of 0.002 in the 1000 Genomes Project. Subsequently, the ClinVar database reported that the c.68-7T $>\mathrm{A}$ variant (ClinVar variation ID: 52187) was classified as likely benign by Invitae (SCV000073064), benign by GeneDx (SCV000108635.3), and benign by the Sharing Clinical Reports Project (SCRP) on behalf of Myriad (SCV000109181.3, initially classified as a VUS and updated in 2012). Through personal communication with Myriad, we also confirmed that they had reclassified the variant to a polymorphism. The variant was also found to co-occur with a pathogenic BRCA2 mutation. ${ }^{23}$ Taken together, there was sufficient evidence to reclassify the variant to benign. Following these reclassification events, the laboratory was contacted by a clinician who had enrolled a patient in a clinical trial based on having received a pathogenic result relating to this variant in 2010, but who had subsequently received a negative report from another testing laboratory. A revised report was issued based on the above benign classification change and the patient was subsequently removed from the clinical trial. Two other individuals who had received a report with a pathogenic classification for this variant were identified and the referring clinicians were recontacted and offered a revised report.

\section{Time between reclassification events}

Prior to 2012, variant data was not consistently captured. The initial identification dates of variants seen prior to 2012 are not reported here. Of the 150 reclassified variants, 122 were first identified before 2012. These 122 variants were reclassified on average 675 days after the establishment of the AMDL variant database. The remaining 28 reclassified variants that were initially identified after the establishment of the database were reclassified on average 456 days after their first identification. For the 25 variants that were reclassified twice, the second reclassification occurred on average 180 days after the first reclassification. One variant was reclassified three times; the third reclassification occurred 102 days after the second reclassification.

\section{Comparison to other submitters in ClinVar}

There were discordances between the AMDL submission to ClinVar and other submitters' assertions for 40.4\% (488/1209) of the variants seen by AMDL (Table 1). For 24.0\% (117/488) of these variants, the discordance between submissions was only a difference in confidence, i.e., between likely pathogenic and pathogenic (45 variants), or between likely benign and benign (72 variants).
Table 1 Number of $B R C A 1 / 2$ variants that AMDL had submitted to ClinVar for which there were discordant submissions

Number of variants with discordant

ClinVar submissions (total $n=488$ )

\begin{tabular}{|c|c|}
\hline \multicolumn{2}{|c|}{ Discrepancy across two ACMG/AMP levels } \\
\hline Likely Benign/Benign & $14.8 \%(72 / 488)$ \\
\hline $\begin{array}{l}\text { Likely Pathogenic/ } \\
\text { Pathogenic }\end{array}$ & $9.2 \%(45 / 488)$ \\
\hline \multicolumn{2}{|c|}{ Discrepancy across three ACMG/AMP levels } \\
\hline $\begin{array}{l}\text { Benign/Likely Benign/ } \\
\text { VUS }\end{array}$ & $68.6 \% \%(335 / 488)$ \\
\hline $\begin{array}{l}\text { Pathogenic/Likely } \\
\text { Pathogenic/VUS }\end{array}$ & $6.1 \% \%(30 / 488)$ \\
\hline \multicolumn{2}{|c|}{ Discrepancy across five ACMG/AMP levels } \\
\hline $\begin{array}{l}\text { Pathogenic/VUS/Likely } \\
\text { Benign/Benign }\end{array}$ & $0.6 \%(3 / 488)$ \\
\hline \multicolumn{2}{|c|}{ Different classification system } \\
\hline Pathogenic/Risk Factor & $0.6 \%(3 / 488)$ \\
\hline
\end{tabular}

ACMG/AMP American College of Medical Genetics and Genomics/Association for Molecular Pathology, ADML Advanced Molecular Diagnostics Laboratory, VUS variant of uncertain significance.

For $74.4 \%(365 / 488)$ of the variants, the discordance between submissions was across three classification levels. For $91.8 \%$ (335/365) variants, the discordance was between VUS/likely benign/benign, and for $8.2 \%$ (30/365) the discordance was between or VUS/likely pathogenic/pathogenic. For $28.2 \%(103 / 365)$ of these variants, only BIC had classified the variant as a VUS, while all other submitters had classified the variant as likely benign/benign or likely pathogenic/pathogenic. Notably, BIC is no longer updated and does not represent up-to-date variant classification information.

For $0.4 \%(2 / 488)$ of the variants, while all other submissions were benign/likely benign/VUS, GeneReviews (one variant) or OMIM (one variant) classified the variant as pathogenic. Of note, OMIM has contributed hundreds of variants to ClinVar that are currently labeled as pathogenic, though these assertions are out of date and not based on a thorough review of evidence.

For $0.2 \%(1 / 488)$, there was a major conflict between submissions. For this variant, BRCA1 c.594-2A $>$ C (ClinVar variation ID: 37686), earlier submitters classified the variant as pathogenic (SCV000145639.1, SCV000187727.1), and later submitters classified it as a VUS (SCV000591290.1, SCV000219194.4, SCV000586870.1, SCV000077080.8), likely benign (SCV000210077.12, SCV000266035.1), and benign (SCV000577983.1), which is now the variant's overall status in ClinVar. This example further illustrates that a variant's classification may change over time.

Finally, for $0.6 \%(3 / 488)$ of the variants, OMIM classified the variants as "risk factors," whereas AMDL and all other submitters classified them as pathogenic. 
For these 488 variants, there were 2111 submissions that were discordant with AMDL submissions. All AMDL submissions were annotated in ClinVar as "criteria provided, single submitter." For $72.3 \%$ (1526/2111), the conflicting submission was also annotated as "criteria provided, single submitter." For $24.0 \%$ (507/2111), the conflicting submission was annotated as was "no assertion criteria provided." Finally, for $3.7 \%(78 / 2111)$ of the submission pairs, the other submission was annotated "reviewed by expert panel."

\section{DISCUSSION}

There were $1209 B R C A 1 / 2$ variants in the AMDL database between 1 January 2012 and 18 August 2017. During this period, $32.9 \%(398 / 1209)$ of the variants were reassessed and $12.4 \%(150 / 1209)$ were reclassified. Interestingly, $13.4 \%$ (146/ 1086) of the variants identified prior to 2016 were reclassified, while only $3.3 \%(4 / 123)$ of the variants identified in 2016 or later were reclassified. This may in part reflect the implementation of the 2015 ACMG/AMP guidelines for variant interpretation, ${ }^{6}$ and the use of population databases such as ExAC and gnomAD.

Our results are in line with other findings that the majority of reclassifications are downgrades. ${ }^{15,24-27}$ Our overall rate of reclassification $(12.4 \%, 150 / 1209)$ is similar to that reported by Turner et al. (2018), who found that 15\% (142/943) of variants in hereditary cancer genes seen in their patients were reclassified between 2012 and $2017 .{ }^{25}$ However, our reclassification rate is higher than that reported by Mersch et al. (2018), who found $6.4 \%(2868 / 44,777)$ of variants in cancer genes were reclassified between 2006 and $2018 .{ }^{27}$ Our rate of VUS reclassification $(14.7 \%, 67 / 453)$ is higher than the rates identified by both Macklin et al. (11.3\%, 30/266) and Mersch et al. $(7.7 \%, 2048 / 26,670)$, and in line with their findings that the majority of reclassified VUSs are downgraded. ${ }^{26,27}$ While only a minority of VUSs are likely to be reclassified to pathogenic, it is nevertheless important to not assume that all VUSs are benign. ${ }^{28}$ Periodic reassessment of VUSs could lead to reclassification and alleviate ambiguity for clinicians and patients. The interval over which to periodically reassess variants is an open debate, as is whether laboratories have a responsibility to routinely reinterpret variants. Our findings suggest that that reassessment every two years may be appropriate, if feasible. However, given the volume of variants that are seen by individual laboratories, it is challenging to reevaluate numerous variants at a given time point. Information technology approaches and secure data sharing platforms may facilitate automated updates to variant interpretations and reporting. ${ }^{4,19,29,30}$

The establishment of data sharing platforms such as ClinVar and the COGR have been instrumental for allowing laboratories to identify and compare areas of discrepancy. 5,7 The proportion of variants for which there were discordant submissions between AMDL and other submitters in ClinVar $(40.4 \%)$ is similar to the discordance rate found among Canadian laboratories participating in the COGR, where the overall rate of discordance of $B R C A 1 / 2$ variant classifications was $38.9 \%$ when using the 5-tier ACMG/AMP classification system. ${ }^{7}$ For laboratories participating in the COGR, data sharing and consensus building reduced variant discordance from $38.9 \%$ to $30.7 \%$ using the 5-tier AMCG/AMP classification scheme, from $26.7 \%$ to $14.2 \%$ using a 3-tier classification scheme, and from $5.0 \%$ to $0.9 \%$ using a 2 -tier scheme. ${ }^{7}$ Data sharing has promise to improve consensus between laboratories; experts urge that all laboratories share data to improve the quality and clinical utility of variant interpretations. ${ }^{5,7}$ The discordances that we identified in ClinVar reflect the evolving nature of variant interpretation. Classifications can change over time as new evidence emerges, but older assertions of variant pathogenicity can remain in databases. Additionally, we identified discordances due to submissions from the BIC database, which is no longer updated, and OMIM, which has contributed many false positive variant calls to ClinVar. As with most variant databases, false positive and negative variant classifications are present, and data derived from these sources should not be taken at face value.

A further challenge is that variant reporting strategies differ between laboratories. For instance, laboratories may differ in how they report ACMG/AMP levels. Some laboratories may report a likely pathogenic variant as a positive result, while others, like AMDL, report it as inconclusive. In addition, laboratories may also provide a result based on case-level data, e.g., an inconclusive result may be issued if a pathogenic variant is identified in a gene that may not be definitively associated with the reason for referral. Determining whether a variant is pathogenic should be independent of interpreting the cause of a disease in a given patient. ${ }^{6}$ Once a pathogenic variant is identified, drawing case-level conclusions about whether the variant explains a patient's phenotype presents additional challenges. ${ }^{31}$

Between 2012 and 2017, our laboratory has seen a clinically significant reclassification in a small number of cases. In this time period, only $0.3 \%$ (4/1209) of variants moved from inconclusive (likely pathogenic, VUS, and likely benign) to positive (pathogenic), and $0.08 \%$ (1/1209) moved from positive to inconclusive. Changes between inconclusive and negative (benign) would not necessarily have impacted clinical care. The example of the BRCA2 c.68-7T $>$ A variant described in the results illustrates how multiple reclassification events over a number of years may affect a single variant and have downstream clinical implications. Periodic variant reassessment is important to ensure that patients' clinical management is based on up-to-date genetic information.

As new knowledge emerges about genetic variants, so do the complexities of how to recontact patients. At present, laboratories typically establish their own policies as to how best to handle recontacting patients about changes in variant reclassification. In Ontario, it is often up to the patient and/or physician to recontact the laboratory to obtain new information on a variant. This is generally understood by the laboratory and the referring clinicians in the province. AMDL makes every effort to recontact clinics if variant classifications change the final result of the report and if such a result may 
alter clinical care, such as reclassifications from inconclusive to positive or vice versa. However, this may differ between laboratories. There is currently variation in how recontacting patients to report updated results occurs within and across jurisdictions. ${ }^{32,33}$ New guidelines state that while recontacting patients is desirable when results have clinical or personal utility, there is no duty to recontact. ${ }^{34}$ Tracking and selecting patients, as well as reinterpreting and following up on updated genetic test results, can be resource-intensive. ${ }^{4,35}$ This may pose practical barriers to recontacting, given that laboratories typically do not receive funding dedicated to these activities. Proposed solutions include the use of databases and information technology, such as applications to automate reporting of reclassified variants to clinicians. ${ }^{29}$ There is a need for more evidence on patient and health care system outcomes related to recontacting patients with updated genetic results, to inform future policies about recontacting patients. $^{36}$

A limitation to this study is that not all variants in the database were reassessed. Overall rates of reclassification may have been higher had more variants been reassessed. This study represents a relatively small number of variants and consists of data from one laboratory with policies that may differ from other laboratories, limiting the generalizability of these results. Despite these limitations, this study offers insight on the frequency, type, and time between variant reclassifications in the $B R C A 1 / 2$ genes, and discordances in $B R C A 1 / 2$ variant interpretations in ClinVar. Future research should address the impact of variant reclassifications on patients and the health care system more broadly.

\section{SUPPLEMENTARY INFORMATION}

The online version of this article (https://doi.org/10.1038/s41436019-0493-2) contains supplementary material, which is available to authorized users.

\section{ACKNOWLEDGEMENTS}

This work was funded by the government of Canada through Genome Canada, the Ontario Genomics Institute (OGI-070), and Can-SHARE. The Can-SHARE project is supported by Genome Quebec; Genome Canada; the government of Canada; the Ministère de l'Économie, Innovation et Exportation du Québec; and the Canadian Institutes of Health Research (fund 141210). This study was supported by the Ontario Ministry of Health and Long-term Care. C.M. received support from the Research Training Centre at St. Michael's Hospital, the Canadian Institutes of Health Research (FRN 160968), and a studentship funded by the Canadian Centre for Applied Research in Cancer Control (ARCC). ARCC receives core funding from the Canadian Cancer Society (grant 2015-703549).

\section{DISCLOSURE}

The authors declare no conflicts of interest.
Publisher's note: Springer Nature remains neutral with regard to jurisdictional claims in published maps and institutional affiliations.

\section{REFERENCES}

1. Amendola LM, Jarvik GP, Leo MC, et al. Performance of ACMG-AMP variant-interpretation guidelines among nine laboratories in the Clinical Sequencing Exploratory Research Consortium. Am J Hum Genet. 2016;98:1067-1076

2. Walsh R, Thomson KL, Ware JS, et al. Reassessment of Mendelian gene pathogenicity using 7,855 cardiomyopathy cases and 60,706 reference samples. Genet Med. 2017;19:192-203

3. Yang S, Lincoln SE, Kobayashi Y, Nykamp K, Nussbaum RL, Topper S. Sources of discordance among germ-line variant classifications in ClinVar. Genet Med. 2017;19:1118-1126.

4. Aronson SJ, Clark EH, Varugheese M, Baxter S, Babb LJ, Rehm HL. Communicating new knowledge on previously reported genetic variants. Genet Med. 2012;14:713-719.

5. Rehm HL. A new era in the interpretation of human genomic variation. Genet Med. 2017;19:1092-1095.

6. Richards S, Aziz N, Bale S, et al. Standards and guidelines for the interpretation of sequence variants: a joint consensus recommendation of the American College of Medical Genetics and Genomics and the Association for Molecular Pathology. Genet Med. 2015;17:405-424.

7. Lebo MS, Zakoor KR, Chun K, et al. Data sharing as a national quality improvement program: reporting on BRCA1 and BRCA2 variantinterpretation comparisons through the Canadian Open Genetics Repository (COGR). Genet Med. 2018;20:294-302.

8. Balmana J, Digiovanni L, Gaddam P, et al. Conflicting interpretation of genetic variants and cancer risk by commercial laboratories as assessed by the Prospective Registry of Multiplex Testing. J Clin Oncol. 2016;34: 4071-4078

9. Vail PJ, Morris B, van Kan A, et al. Comparison of locus-specific databases for BRCA1 and BRCA2 variants reveals disparity in variant classification within and among databases. J Community Genet. 2015;6:351-359.

10. Lincoln SE, Yang S, Cline MS, et al. Consistency of BRCA1 and BRCA2 variant classifications among clinical diagnostic laboratories. JCO Precis Oncol 2017;1. Epub 2017 Apr 11.

11. Nelson HD, Fu R, Goddard K, et al. Risk Assessment, Genetic Counseling, and Genetic Testing for BRCA-Related Cancer: Systematic Review to Update the U.S. Preventive Services Task Force Recommendation. Ann Intern Med. 2014;160:255-266.

12. Antoniou A, Pharoah PD, Narod S, et al. Average risks of breast and ovarian cancer associated with BRCA1 or BRCA2 mutations detected in case Series unselected for family history: a combined analysis of 22 studies. Am J Hum Genet. 2003;72:1117-1130.

13. Chen S, Parmigiani G. Meta-analysis of BRCA1 and BRCA2 penetrance. J Clin Oncol. 2007;25:1329-1333.

14. Eccles DM, Mitchell G, Monteiro AN, et al. BRCA1 and BRCA2 genetic testing-pitfalls and recommendations for managing variants of uncertain clinical significance. Ann Oncol. 2015;26:2057-2065.

15. Murray ML, Cerrato F, Bennett RL, Jarvik GP. Follow-up of carriers of BRCA1 and BRCA2 variants of unknown significance: variant reclassification and surgical decisions. Genet Med. 2011;13:998-1005.

16. Finch $A$, Wang $M$, Fine $A$, et al. Genetic testing for BRCA1 and BRCA2 in the Province of Ontario. Clin. Gen. 2016;89:304-311.

17. Ontario Ministry of Health and Long Term Care. MOHLTC Criteria for Breast and Colon Cancer Screning. Mount Sinai Hospital 2007. https:// www.mountsinai.on.ca/care/pathology/laboratory-forms-andrequisitions/MOHLTC \%20C riteria\%20for\%20Breast\%20and\%20Colon \%20Cancer\%20Screening.pdf. Accessed 8 April 2019.

18. Duzkale $\mathrm{H}$, Shen J, McLaughlin $\mathrm{H}$, et al. A systematic approach to assessing the clinical significance of genetic variants. Clin Genet. 2013;84:453-463.

19. Lerner-Ellis J, Wang M, White S, Lebo MS, Canadian Open Genetics Repository Group. Canadian Open Genetics Repository (COGR): a unified clinical genomics database as a community resource for standardising and sharing genetic interpretations. J Med Genet. 2015; 52:438-445.

20. Richards CS, Bale S, Bellissimo DB, et al. ACMG recommendations for standards for interpretation and reporting of sequence variations: revisions 2007. Genet Med. 2008;10:294-300. 
21. Landrum ML, Lee JM, Benson M, Brown GR, Chao C, Chitipiralla S, et al. ClinVar: improving access to variant interpretations and supporting evidence. Nucleic Acids Res. 2018;46:D1062-7.

22. Lek M, Karczewski KJ, Minikel EV, et al. Analysis of protein-coding genetic variation in 60,706 humans. Nature. 2016;536:285-291.

23. Muller $D$, Rouleau $E$, Schultz I, et al. An entire exon 3 germ-line rearrangement in the BRCA2 gene: pathogenic relevance of exon 3 deletion in breast cancer predisposition. BMC Med Genet. 2011;12:121.

24. Kast $K$, Wimberger $P$, Arnold $N$. Changes in classification of genetic variants in BRCA1 and BRCA2. Arch Gynecol Obstet. 2018;297:279-280.

25. Turner SA, Rao SK, Morgan RH, Vnencak-Jones CL, Wiesner GL. The impact of variant classification on the clinical management of hereditary cancer syndromes. Genet Med. 2019;21:426-430.

26. Macklin S, Durand N, Atwal P, Hines S. Observed frequency and challenges of variant reclassification in a hereditary cancer clinic. Genet Med. 2018;20:346-350.

27. Mersch J, Brown N, Pirzadeh-Miller $S$, et al. Prevalence of variant reclassification following hereditary cancer genetic testing. JAMA. 2018;320:1266-1274.

28. Weck KE. Interpretation of genomic sequencing: variants should be considered uncertain until proven guilty. Genet Med. 2018;20:291-293.

29. Aronson SJ, Clark EH, Babb LJ, et al. The Genelnsight Suite: a platform to support laboratory and provider use of DNA-based genetic testing. Hum Mutat. 2011:32:532-536.
30. Bean $\sqcup$, Tinker SW, da Silva C, Hedge MR. Free the data: one laboratory's approach to knowledge-based genomic variant classification and preparation for EMR integration of genomic data. Hum Mutat. 2013;34: 1183-1188.

31. Strande NT, Brnich SE, Roman TS, Berg JS. Navigating the nuances of clinical sequence variant interpretation in Mendelian disease. Genet Med. 2018;20:918-926.

32. Chisholm C, Daoud H, Ghani M, et al. Reinterpretation of sequence variants: one diagnostic laboratory's experience, and the need for standard guidelines. Genet Med. 2018;20:365-368.

33. Sirchia F, Carrieri D, Dheensa $S$, et al. Recontacting or not recontacting? A survey of current practices in clinical genetics centres in Europe. Eur Hum Genet. 2018;26:946-954.

34. Carrieri D, Howard HC, Benjamin C, et al. Recontacting patients in clinical genetics services: recommendations of the European Society of Human Genetics. Eur J Hum Genet. 2018;27:169-182.

35. Beunders G, Dekker M, Haver O, Meijers-Heijboer HJ, Henneman L. Recontacting in light of new genetic diagnostic techniques for patients with intellectual disability: Feasibility and parental perspectives. Eur J Med Genet. 2018;61:213-218

36. Bombard $Y$, Mighton $C$ Recontacting clinical genetics patients with reclassified results: equity and policy challenges. Eur J Hum Genet. 2019:27:505-506. 